\title{
WG5 summary: direct CP violation in B decays
}

\author{
Malcolm John* \\ University of Oxford \\ E-mail: malcolm.johnephyiscs.ox.ac.uk
}

Thomas Latham

University of Warwick

E-mail: t.latham@warwick.ac.uk

Rukmani Mohanta

University of Hyderabad

E-mail: rmspeuohyd.ernet.in

These proceedings summarise new results shown at the 2016 CKM workshop in Working Group 5. Time-integrated charge asymmetry measurements in $B \rightarrow D h$ and charmless $b$-hadron decays are core themes of the group but several ancillary topics are reported. Vital to understanding $B \rightarrow D^{0} K$ charge asymmetries in multibody $D^{0}$ decays is knowledge of the $C P$ content of the $D^{0}$ decay and two important developments are described. Also reported is a number of searches for new hadronic $B$ decay signatures. Experimental results are dominated by $\mathrm{LHCb}$ at this time though, results from Belle are still forthcoming.

9th International Workshop on the CKM Unitarity Triangle

28 November - 3 December 2016

Tata Institute for Fundamental Research (TIFR), Mumbai, India

${ }^{*}$ Speaker. 


\section{Introduction}

These proceedings summarise the the study of direct $C P$ violation in hadronic $B$ decays as presented at the conference. The results are fall into three categories: measurements of $C P$ observables from the $B \rightarrow D K$ family of decays; studies of direct $C P$ violation in charmless decays; searches for new hadronic modes which may be later studied in the context of $C P$ violation.

There is an emphasis on open charm decays of $B^{+}$and $B^{0}$ mesons to the $D^{(*)} K^{(*)}$ final states because these decays have tree-level sensitivity to the Unitarity Triangle angle $\gamma$. The importance of the tree-level sensitivity is that these decays suffer negligible corrections from higher-order loop contributions. In contrast to the charmed case, $C P$-violation in charmless decays requires the interference of tree and loop (penguin) amplitudes. The separation of the hadronic (strong) parameters from the CKM (weak) information is not trivial.

2. $B \rightarrow D^{0} K^{(*)}$

The sensitivity to the $C P$-violating weak phase of the CKM matrix is achieved in $B \rightarrow D^{0} K^{(*)}$ decays because the rates for $B$ and $\bar{B}$ decays depend on $\gamma=\arg \left(-V_{u d} V_{u b}^{*} / V_{c d} V_{c b}^{*}\right)$, which is closely related to the CKM phase. Decays of this type are dominated by $b \rightarrow c \bar{u} s$ tree-level transitions but receive a significant contribution from $b \rightarrow u \bar{c} s$ transitions, also a tree decay. The interference of these amplitudes allows sensitivity to the phases between them which can be further separated into $C P$-violating and $C P$-conserving parts. The $C P$-violating phase is that of the weak force where the $C P$-conserving part is associated with the dynamics of the strong forces in the formation of the mesons. Inspection of the contributing CKM elements identifies the weak phase in $B \rightarrow D^{0} K^{(*)}$ decays as $\gamma \approx \arg \left(-V_{u b}^{*} / V_{c b}^{*}\right)$. To be sensitive to interference effects the charm meson must be reconstructed in final state accessible (with similar magnitude) from both $D^{0}$ and $\bar{D}^{0}$. Conceptually this is simplest where the charm meson is reconstructed in a $C P$ eigenstate as this is equally accessible to both $D^{0}$ and $\bar{D}^{0}$ (in the absence of direct $C P$ violation in the charm decay) as advocated in the GLW method [1]. It can be shown that the rates for $B^{+}$and $B^{-}$thus differ,

$$
\Gamma\left(B^{ \pm} \rightarrow D_{C P} K^{ \pm}\right)=\left|1+r_{B} e^{i\left(\delta_{B} \pm \gamma\right)}\right|^{2}=1+r_{B}^{2}+2 r_{B} \cos \left(\delta_{B} \pm \gamma\right),
$$

where the magnitude of the $b \rightarrow u \bar{c} s$ amplitude compared to that of the $b \rightarrow c \bar{u} s$ transition is the factor $r_{B}$ and the $C P$-conserving phase between the two amplitudes is labelled $\delta_{B}$. The ADS method [2] is a generalisation that can include non- $C P$ eigenstate decays of the $D^{0}$ by included a factor relating $\bar{D}^{0}$ and $D^{0}$ to a common final state, $r_{D} e^{-i \delta_{D}}$. In the charm meson decay only a $C P$-conserving phase is needed as $C P$-violating effects are neglected; the minus sign needed by convention. For example $K^{-} \pi^{+}$which doubly-Cabibbo-suppressed in the case that it comes from a $\bar{D}^{0}$ but favoured from a $D^{0}$. By choosing combinations where the charge of the kaon from the $B$ and $D$ are of opposite charge, the two interfering amplitudes represented in Eq. 2.1 become similar in size and thus much larger interference effects (asymmetries) are possible. The $B^{ \pm}$decay rate becomes,

$$
\Gamma\left(B^{ \pm} \rightarrow\left[\pi^{ \pm} K^{\mp}\right]_{D} K^{ \pm}\right)=\left|r_{D} e^{-i \delta_{D}}+r_{B} e^{i\left(\delta_{B} \pm \gamma\right)}\right|^{2}=r_{D}^{2}+r_{B}^{2}+2 \kappa r_{D} r_{B} \cos \left(\delta_{B}-\delta_{D} \pm \gamma\right),
$$

where $\kappa=1$ for two-body $D$-decays. Note that in the case of a $C P$-eigenstate decay, $r_{D}=1$ and $\delta_{B}=0$ and Eq. 2.2 reverts to Eq. 2.1. This formula generalises to three- or four-body final states 
by a non-unity value of the dilution parameter, $\kappa$, in the interference term of Eq. 2.2. In the special cases that a self-conjugate charm decay is used, notably $D^{0} \rightarrow K_{\mathrm{S}}^{0} \pi^{+} \pi^{-}$, the dilution factor is approximately zero and so, taken inclusively, the $C P$ information washes out. In this case, it is preferable to measure $C P$ asymmetries across the $D$ decay Dalitz plane with the GGSZ method [3].

\subsection{Combination of results for $\gamma$}

With so many techniques and no overall golden mode for the measurement of $\gamma$ in $B \rightarrow D^{0} K^{(*)}$ decays, a statistical combination is made. This inference must take into account correct treatment of nuisance parameters and consideration for coverage. Two techniques are common,

- A Frequentist statistical approach starts from a likelihood function, built from the product of probability density functions (PDFs). The PDFs are calculated from the experimental observables and their uncertainties assuming that these uncertainties describe a multidimensional Gaussian distribution.

- A Bayesian statistical approach calculates probability regions in the multi-parameter space by finding the highest posterior probability density, integrating over the nuisance parameters. The posterior probability density is related to the the likelihood by the prior probability of the parameters. In all work described here, the prior probability are assumed to be uniform.

The most precise measurements of the $C P$ observables from $B \rightarrow D K$ decays come from $\mathrm{LHCb}$ and that collaboration have performed a statistical inference using their published results [4]. Using results from the ADS/GLW and GGSZ analyses of $B^{+} \rightarrow D K^{+}$decays and $B^{0} \rightarrow D K^{0}$ decays, plus time-dependent asymmetries in $B_{s}^{0} \rightarrow D_{s}^{+} K^{-}$decays, LHCb infer values of $\gamma$ using both a Frequentist and Bayesian approach,

\section{$\left(72.2_{-7.3}^{+6.8}\right)^{\circ}$ Frequentist LHCb and $\left(70.3_{-7.9}^{+7.1}\right)^{\circ}$ Bayesian LHCb,}

which are considered compatible, given the assumptions made by the competing techniques.
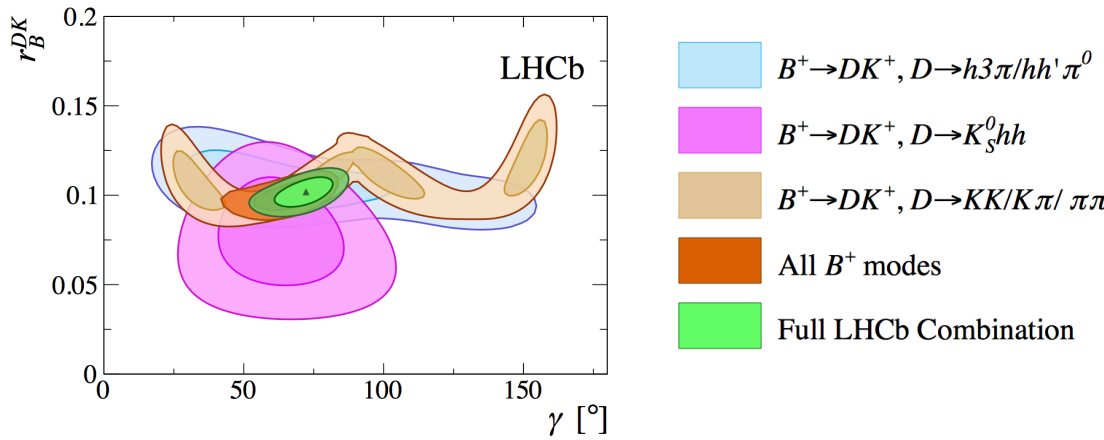

Figure 1: Profile likelihood contours for $r_{B} v s . \gamma$, where $r_{B}$ is the ratio of suppressed to favoured amplitudes contributing to $B^{+} \rightarrow D K^{+}$decays. The measurements that contribute to each contour are described in the legend. The full frequentist $\mathrm{LHCb}$ combination is shown in green. In each case the dark and light regions show the intervals containing $68.3 \%$ and $95.5 \%$ respectively.

The profile likelihood contours for $B^{+} \rightarrow D K^{+}$decays are shown in Fig. 1. The full LHCb combination illustrates the important contribution from $B^{+} \rightarrow D K^{+}$modes. A further combination 
which includes $C P$ observables in the ADS/GLW analyses of $B^{+} \rightarrow D \pi^{+}$decays with the effect of $D$-mixing properly taken into account shows negligible improvement in the uncertainty of $\gamma$. Dedicated proceedings describe the contributing LHCb analyses from $B \rightarrow D^{(*)} K^{*}$ decays [5] and $B \rightarrow D h h^{\prime}$ decays [6].

Global combinations, which include legacy measurements from the $B$-factories are also available from the CKMFitter [7] and UTFit [8, 9] groups with updates presented at conference; these results are seen to be similar to, and dominated by, the LHCb measurements,

$\left(72.2_{-5.8}^{+5.3}\right)^{\circ}$ Global Frequentist (CKMFitter) and $(70.5 \pm 5.7)^{\circ}$ Global Bayesian (UTFit) .

$2.2 B^{+} \rightarrow D K^{*+}$

An important new result, presented for the first time from LHCb, is a preliminary ADS/GLW analysis of $B^{-} \rightarrow D K^{*-}$ [10]. This is the first time that this mode has been reconstructed at a hadron collider though results have been available from the Babar for some time [11]. The analysis
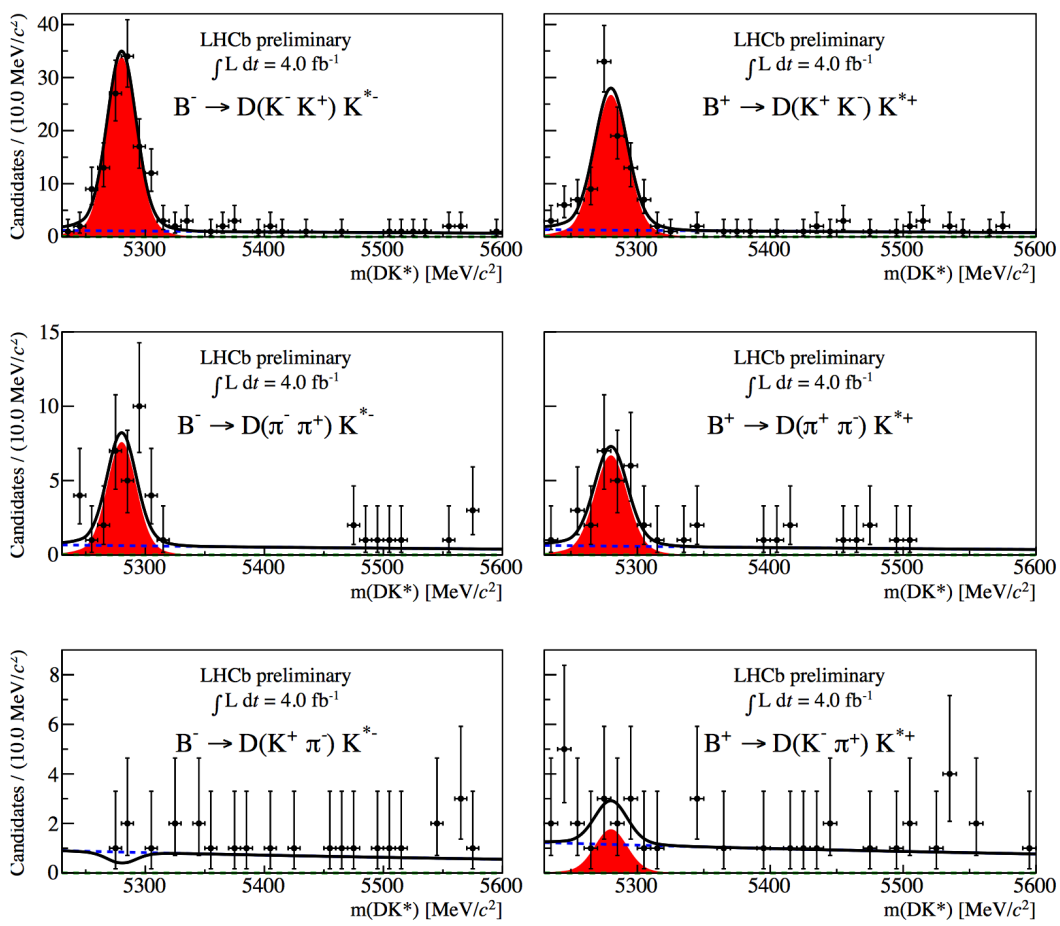

Figure 2: Invariant mass distributions to $B^{-} \rightarrow D K^{*-}$ decays (left) and $B^{+} \rightarrow D K^{*+}$ decays (right) from LHCb. From top to bottom the $D^{0}$ decay modes are $K^{+} K^{-}, \pi^{+} \pi^{-}$and suppressed $K \pi$ combinations. The signal component is represented by the red area.

reconstructs $K^{*-} \rightarrow K_{\mathrm{S}}^{0} \pi^{-}$which means the number of events with respect to an analysis of $B^{-} \rightarrow$ $D K^{-}$decays is lower by the branching fraction of $K^{*-} \rightarrow\left[\pi^{+} \pi^{-}\right]_{K_{\mathrm{S}}^{0}} \pi^{-}\left(\approx \frac{2}{3}\right)$ and the efficiency of reconstructing and selecting a $K_{\mathrm{S}}^{0}$ in the experiment $(\approx 12 \%$ for $\mathrm{LHCb})$. The $D K^{*+}$ final state has the advantage that there is no large misidentified physics background, like $B^{-} \rightarrow D^{0} \pi^{-}$in the case of $B^{-} \rightarrow D^{0} K^{-}$. The $C P$ observables are ratios of branching fractions and charge asymmetries,

$$
R_{C P+}=\quad 2 \frac{\Gamma\left(B^{-} \rightarrow D_{C P} K^{*-}\right)+\Gamma\left(B^{+} \rightarrow D_{C P} K^{*+}\right)}{\Gamma\left(B^{-} \rightarrow D^{0} K^{*-}\right)+\Gamma\left(B^{+} \rightarrow \bar{D}^{0} K^{*+}\right)} \quad=1.21 \pm 0.10,
$$




$$
\begin{aligned}
& A_{C P+}=\frac{\Gamma\left(B^{-} \rightarrow D_{C P} K^{*-}\right)-\Gamma\left(B^{+} \rightarrow D_{C P} K^{*+}\right)}{\Gamma\left(B^{-} \rightarrow D_{C P} K^{*-}\right)+\Gamma\left(B^{+} \rightarrow D_{C P} K^{*+}\right)}=0.11 \pm 0.07, \\
& R_{\mathrm{ADS}}=\frac{\Gamma\left(B^{-} \rightarrow\left[\pi^{-} K^{+}\right]_{D} K^{*-}\right)+\Gamma\left(B^{+} \rightarrow\left[\pi^{+} K^{-}\right]_{D} K^{*+}\right)}{\Gamma\left(B^{-} \rightarrow D^{0} K^{*-}\right)+\Gamma\left(B^{+} \rightarrow \bar{D}^{0} K^{*+}\right)}=0.003 \pm 0.004 .
\end{aligned}
$$

The observables with the $C P$ subscript are taken from the weighted sum of the results in the $K^{+} K^{-}$ and $\pi^{+} \pi^{-} D$-meson modes. $R_{\mathrm{ADS}}$ is the rate of the suppressed mode, where the kaon from the $D$ decay is of opposite charge to that of the $K^{*}$, compared to the favoured mode. These suppressed mode data are shown in the third row of Fig. 2 from which an intriguing charge asymmetry is seen. As non-resonant $K_{\mathrm{S}}^{0} \pi^{-}$can contribute non-negligibly to the signal due to the necessarily large mass window of the $K^{*-}$ selection. This introduces a dependence on a coherence factor $\kappa$ as described in Eq. 2.2, estimated to be $0.95 \pm 0.06$ within the $K^{*-}$ mass and helicity window requirements. The parameter $\kappa$ is estimated by generating many amplitude models for $B \rightarrow D K_{\mathrm{s}}^{0} \pi$ decays consisting of resonant components whose relative amplitudes and phases are varied within limits according to branching ratio measurements.

\section{$2.3 B$-factories and $\gamma$}

Though most $B \rightarrow D K$ analyses were first made at Belle and Babar, the large LHCb dataset means that experiment's results currently provide for most information on $\gamma$. The $B$-factory data nevertheless continues to make new contributions, especially in with modes that involve neutral particles. It is known that a useful addition to the $B \rightarrow D K$ suite of analyses is that of $B^{+} \rightarrow D^{*} K^{+}$ decays with $D^{* 0} \rightarrow D^{0} \pi^{0}$ and $D^{* 0} \rightarrow D^{0} \gamma$. Belle report an analysis of this mode using $711 \mathrm{fb}^{-1}$, or 7.72 million $B \bar{B}$ events, with $C P$ observables,

$$
\begin{array}{lrrrr}
R_{C P+}= & 1.19 \pm 0.13 \pm 0.03, & R_{\mathrm{ADS}}\left(D \pi^{0}\right) & = & \left(1.0_{-0.7}^{+0.8}+0.1\right) \% \\
R_{C P-}= & 1.03 \pm 0.13 \pm 0.03, & R_{\mathrm{ADS}}(D \gamma) & = & \left(3.6_{-1.2}^{+1.4} \pm 0.2\right) \% \\
A_{C P+}= & -0.14 \pm 0.10 \pm 0.01, & A_{\mathrm{ADS}}\left(D \pi^{0}\right) & = & +0.4_{-0.1}^{+1.1}+0.2 \\
A_{C P-}= & +0.22 \pm 0.11 \pm 0.01, & A_{\mathrm{ADS}}(D \gamma) & = & -0.5 \pm 0.3 \pm 0.1
\end{array}
$$

A $3.5 \sigma$ signal is claimed for the $D^{* 0} \rightarrow D \gamma$ ADS mode. Full details and implications for $\gamma$ are anticipated soon [12].

From late 2018, the new $e^{+} e^{-} B$-factory, Belle II, starts collecting data. The benefits of the higher efficiency, especially for neutral particles, means Belle II will bring significant competition to $\mathrm{LHCb}$ in the pursuit of degree-level precision on $\gamma$. A comparison of the expected uncertainty on $\gamma$ for LHCb and Belle II is shown in Fig. 3, reproduced from the dedicated proceedings [12]. These projections, based on extrapolating uncertainties from two-body GLW/ADS analyses plus $K_{\mathrm{S}}^{0} \pi^{+} \pi^{-}$GGSZ analyses, suggest that the two experiments will approach statistical uncertainties of less than $2^{\circ}$ within eight years. This prediction is conservative because there are a large number of additional modes that both experiments can exploit.

\subsection{Limitation for precision measurements}

The culmination of $B \rightarrow D^{0} K$ analyses over the next decade will be a precision on $\gamma$ around $1^{\circ}$. At this level, $\gamma$ will be known to a similar precision as with the measurement of the Unitarity Triangle $\beta$ from $B^{0} \rightarrow J / \psi K^{0}$. The theoretical uncertainty on the value of $\gamma$ from $B \rightarrow D K$ decays is far beyond this experimental limit because there is a negligible loop contribution in $B \rightarrow D K$ 

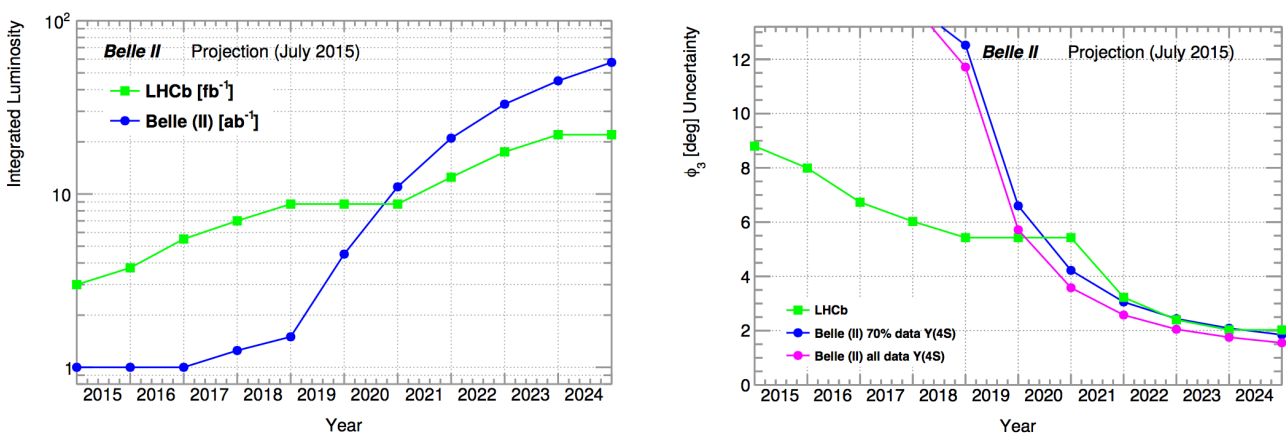

Figure 3: Projected dataset size (left) and projected uncertainty on $\gamma$ (right) as a function of time.

decays and the hadronic parameters can be fitted from data. In recent work the ultimate limitations, from a theoretical point of view, are identified:

- Measurements of $\gamma$ are biased by $D$ mixing effects though these can be entirely corrected for with precise knowledge of the $D$ mixing parameters and the lifetime acceptance [13].

- The $C P$ violation in $K^{0}$ mixing can cause the biases in modes involving a $K_{\mathrm{S}}^{0}$. Uncorrected, the bias is of the order $\varepsilon_{K} / r_{B}$ where $\varepsilon_{K} \approx 2 \times 10^{-3}$ so can be a significant bias for GGSZ analyses with $B \rightarrow D \pi$ decays [14].

- The $C P$-violating phase measured from the interference in $B \rightarrow D K$ decays is not exactly $\gamma$, as defined from the Unitarity Triangle. The difference is due to sub-leading terms that are considered to be without an imaginary component. To correct for this effect requires precise knowledge of the ratio $V_{c d} V_{c s}^{*} / V_{u d} V_{u s}^{*} \sim 2.6 \times 10^{-3}[15]$.

- The irreducible theoretical uncertainty in $\gamma$ comes from the contribution from alternative Feynmann diagrams with different CKM elements. Second-order box diagrams can contribute to the $b \rightarrow c \bar{u} s$ amplitude with different CKM elements (the $b \rightarrow u \bar{c} s$ amplitude is safe) resulting in a limiting theoretical uncertainty $\left|\delta_{\gamma} / \gamma\right| \leq 10^{-7}$ for $B \rightarrow D K$ decays and $\leq 10^{-4}$ in the case of $B \rightarrow D \pi$ decays [16].

In a related study [17], the assumption of no new physics at tree-level is reviewed. It is found that a universal New Physics contribution to tree-level $B$ decays is not excluded by the data. The intrinsic, model-independent, theoretical uncertainty on $\gamma$ in this paradigm is $\left|\delta_{\gamma}\right| \approx 4^{\circ}$ so this speculative possibility will soon be constrained by direct measurement. It is also noted that $B \rightarrow D K$ decays have a built-in test for New Physics in the presumption that the ratio of amplitudes $r_{B}$ is the same for $b$ - and $\bar{b}$-quark transitions. Tests for New Physics should thus retain sensitivity to $r_{B-} \neq r_{B+}$.

\section{Charm inputs for $B \rightarrow D K$ analyses}

The understanding of $C P$-violation in $B \rightarrow D K$ in anything other that the simplest GLW analysis requires external information on the hadronic parameters of the neutral charm decay that is produced from the $B$ decay. As discussed above, the ADS and GLW modes can be generalised to 
three or more particles in the final state by including a dilution parameter. Where the dilution parameter is small, it is not useful to approximate to a two-body and a GGSZ (or GGSZ-like) analysis is used. In this case, the $C P$ content of the $D^{0}$ decay is described by $n\left(c_{i}, s_{i}\right)$ parameters $(1<i<n)$ defined in $n$ bins across the Dalitz space of the multibody $D^{0}$ decay.

$$
c_{i}+i s_{i}=\frac{\int_{i} A_{f}(\mathbf{p}) \bar{A}_{f}(\mathbf{p})^{*} d \mathbf{p}}{\sqrt{\int_{i}\left|A_{f}(\mathbf{p})\right|^{2} d \mathbf{p} \cdot \int_{i}\left|\bar{A}_{f}(\mathbf{p})\right|^{2} d \mathbf{p}}},
$$

where the amplitude for the $\bar{D}^{0}$ going to final state $f$ at point $\mathbf{p}$ on the Dalitz space is $\bar{A}_{f}(\mathbf{p})$. In the GGSZ analysis of $D \rightarrow K_{\mathrm{S}}^{0} \pi^{+} \pi^{-}$, symmetric binning around the $y=x$ diagonal of the Dalitz plot ensures a simple relation between the $C P$-conjugate bins either side of this divide, $c_{-i}=c_{i}, s_{-i}=$ $-s_{i}$. In recent work, these ideas have been expanded to four-body self-conjugate final states.

3.1 $D^{0} \rightarrow \pi^{+} \pi^{-} \pi^{+} \pi^{-}$

In analysis of $B^{+} \rightarrow\left[\pi^{+} \pi^{-} \pi^{+} \pi^{-}\right]_{D} K^{+}$decays by LHCb [18] demonstrates event yields similar to the primary GGSZ mode, $B^{+} \rightarrow\left[K_{\mathrm{S}}^{0} \pi^{+} \pi^{-}\right]_{D} K^{+}$[19]. Furthermore, the overall $C P$-fraction in this mode is only $F_{+}=0.737 \pm 0.028$ [20]. In terms of an overall, total phase-space averaged $c_{0}, s_{0}$, this is $c_{0}=2 F_{+}-1=0.474 \pm 0.056, s_{0}=0.0$, which means that there is a potential factor of two increase in sensitivity with a binning of $c_{i}, s_{i}$ measurements.

A four-body amplitude analysis of $D^{0} \rightarrow \pi^{+} \pi^{-} \pi^{+} \pi^{-}$has recently become completed using data collected by the CLEO-c detector [21]. Prominent contributions are found from the decay modes $D^{0} \rightarrow a_{1}(1260)^{+} \pi^{-}, D^{0} \rightarrow \sigma f_{0}(1370)$ and $D^{0} \rightarrow \rho^{0}(770) \rho^{0}(770)$. From this model, an adaptive binning scheme is developed [22] to find an appropriate granularity to adequately partition the five-dimensional Dalitz space for the purposes of measuring $c_{i}, s_{i}$ for this mode.

Preliminary results for $c_{i}, s_{i}$ in $D^{0} \rightarrow \pi^{+} \pi^{-} \pi^{+} \pi^{-}$decays using CLEO-c quantum-correlated data are also presented. The quantum-correlated technique tags the flavour or $C P$ content of a $D^{0} \rightarrow$ $\pi^{+} \pi^{-} \pi^{+} \pi^{-}$decay from the flavour or $C P$ content of the other neutral $D$ mesons from a $\psi(3770)$ decay. In this preliminary work, only flavour and $C P$ eigenstate tags are used and not the mixed-tag from $D \rightarrow K_{\mathrm{S}}^{0} \pi^{+} \pi^{-}$decays. This means the preliminary result, shown in Fig. 4 gives only $c_{i}$, and not $s_{i}$, information. However, a projection is also shown in the figure of the eventual precision on both ordinates. Once the binning definition and the $c_{i}$ and $s_{i}$ measurements are published, LHCb and Belle II will be able to perform a binned GGSZ-like analysis of $B^{+} \rightarrow\left[\pi^{+} \pi^{-} \pi^{+} \pi^{-}\right]_{D} K^{+}$.

3.2 $D^{0} \rightarrow K_{\mathrm{S}}^{0} \pi \pi \pi^{0}$

A similar analysis is presented for another self-conjugate mode, $D^{0} \rightarrow K_{\mathrm{S}}^{0} \pi \pi \pi^{0}$. This mode has, so far, not been used in analysis of $B \rightarrow D K$ decays, but this important work shows it to have excellent sensitivity to $\gamma$ with sufficient statistics. Three conclusions are drawn: a first measurement of the $C P$ fraction is made from analysis of the CLEO-c dataset, $F_{+}=0.246 \pm 0.018$, the small fraction means this mode approximates as a $C P$-odd final state. Second, an eight-bin scheme is devised around the expected intermediate resonances (in the absence of a full amplitude model) and measurements of the $c_{i}, s_{i}$ parameters are made, again using the CLEO-c data. Third, a sensitivity study of a $B^{-} \rightarrow\left[K_{\mathrm{S}}^{0} \pi^{+} \pi^{-} \pi^{0}\right]_{D} K^{-}$analysis at Belle II concludes that this mode may have a singlemode sensitivity to $\gamma$ of $3.5^{\circ}$ with $50 \mathrm{ab}^{-1}$. This study and the graphical representation of the $c_{i}, s_{i}$ results are shown in Fig. 5. A dedicated proceedings is available [23]. 

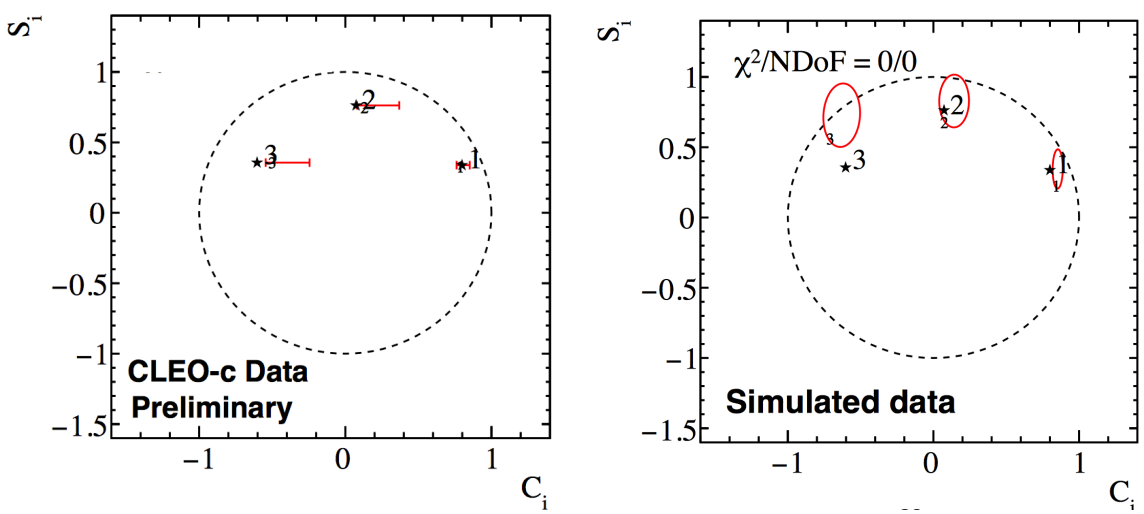

Figure 4: Preliminary measurement of the $C P$ content of $D^{0} \rightarrow \pi^{+} \pi^{-} \pi^{+} \pi^{-}$decays using CLEO-c data (left) and projected accuracy from simulation once the analysis includes $K_{\mathrm{S}}^{0} \pi^{+} \pi^{-}$tags (right). In both cases the black star marks the centre of the bin and the red line(oval) is the measurement(projection).
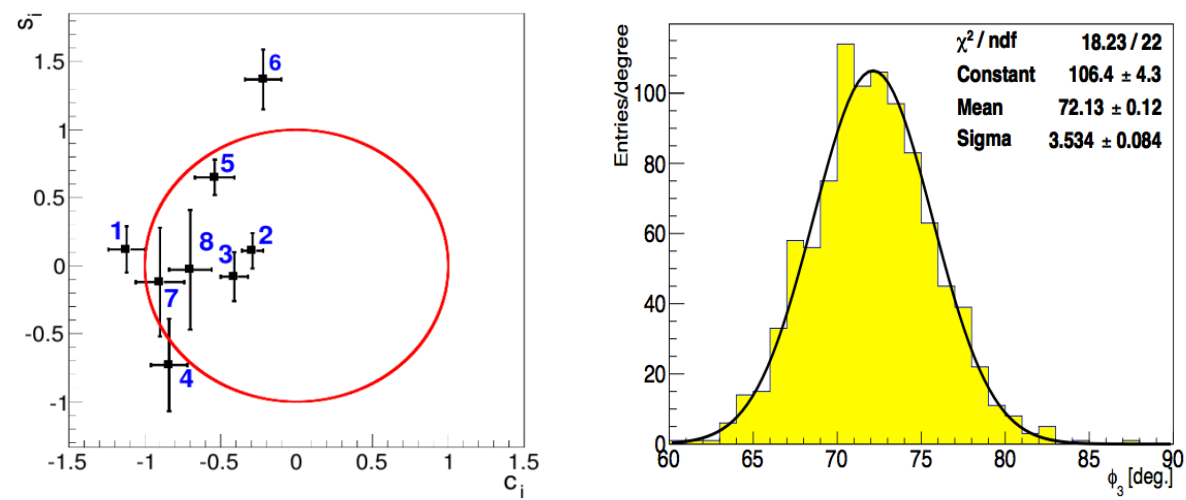

Figure 5: Preliminary measurement of $c_{i}, s_{i}$ from an analysis of $D^{0} \rightarrow K_{\mathrm{S}}^{0} \pi^{+} \pi^{-} \pi^{0}$ decays using CLEO-c data (left) and projected precision on $\gamma$ from this mode with $50 \mathrm{ab}^{-1}$ of Belle II (right).

\section{Direct $C P$ violation in charmless $b$-hadrons}

$C P$ violation in charmless $B^{+}, B^{0}$ and $B_{s}^{0}$ decays arises from the interference of tree and penguin contributions. Extracting CKM information from experimental measurements depends upon the theoretical understanding of the hadronic dynamics. The perturbative calculations for twobody $B$ decays is complete up to $\mathscr{O}\left(\alpha_{s}^{2}\right)[24,25,26]$ and resulting predictions of $C P$ asymmetries agree with experiment [27], though large theoretical uncertainties from long-distance effects persist. Increasingly, multibody decays of $b$-hadrons are being examined by experiments though the associated theory calculation is a challenge.

\subsection{Multibody phenomenology}

The theoretical understanding of multibody charmless decays is hindered by the increased complexity of the multibody kinematics and a larger variety of final states. Some efforts to extend the framework of QCD factorisation to $B$ decays into three light mesons has been made [28] but a full NLO calculation of three-body $B$ decays remains a future challenge. 
A phenomenological study of $B \rightarrow K \pi \pi$ is presented at conference and later published [29]. It combines Belle and Babar analyses for $B^{0} \rightarrow K^{*} \pi$ and $B^{+} \rightarrow K^{*} \pi$ decays in an isospin analysis, which allows to express these decays in terms of CKM parameters and 6 complex hadronic amplitudes: colour allowed tree and penguin; annihilation and colour suppressed tree; colour allowed and colour suppressed electroweak penguin amplitudes. Conclusions are drawn from setting upper bounds on the assumed-small electroweak penguin to make measurements of the Unitarity angle $\alpha$. A conclusion of this work is that the bound on the electroweak penguin amplitude is not precise from the theoretical point of view and doubt is cast on the potential of this method to constrain $\alpha$. The logic is then reversed, drawing conclusions about the hadronic factors by holding CKM-elements to their best fit externally determined values [7]. They find that the Babar analysis of $B^{0} \rightarrow K^{+} \pi^{-} \pi^{0}$ is important in predicting a large contribution from electroweak penguin amplitudes, and conclude that new analysis with more data from LHCb and Belle II is motivated.

\subsection{Confirmation of large $C P$ violation in regions of the $B^{+} \rightarrow K^{+} K^{-} \pi^{+}$Dalitz space}

Belle report an examination of $B^{+} \rightarrow K^{+} K^{-} \pi^{+}$decays that have been shown in a prior $\mathrm{LHCb}$ analysis to exhibit large $C P$ violation in certain regions of the decay phase space [30]. With the whole Belle dataset of $711 \mathrm{fb}^{-1}, 715 \pm 48$ events are selected, and are seen to be concentrated at low $m\left(K^{+} K^{-}\right)$and exhibit a large $C P$ asymmetry, similar to the LHCb data. This result is shown in Fig. 6, which is reproduced from the dedicated proceedings [31].
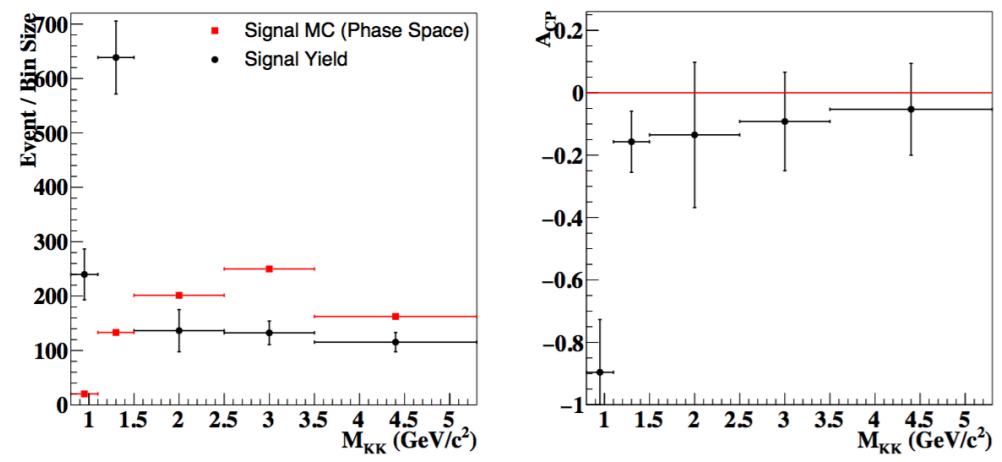

Figure 6: left: $B^{+} \rightarrow K^{+} K^{-} \pi^{+}$event yields as a function $m(K K)$. right: The corresponding asymmetry.

\subsection{Evidence of $C P$ violation in $\Lambda_{b}^{0} \rightarrow p^{+} \pi^{-} \pi^{+} \pi^{-}$}

With $C P$ violation well established in $B$ mesons, it is natural to search for $C P$ asymmetries in $b$-baryons. Large, $\mathscr{O}(20 \%)$ asymmetries are postulated in multibody $\Lambda_{b}^{0}$ decays but are as yet unobserved. To search for $C P$ violation, $\mathrm{LHCb}$ measure $C P$-odd observables by studying asymmetries in a sample of $6646 \pm 105 \Lambda_{b}^{0} \rightarrow p^{+} \pi^{-} \pi^{+} \pi^{-}$decays.

The asymmetries are built from scalar triple products of three of the $\Lambda_{b}^{0}$ daughters in such a way as to form two quantities, $a_{P}^{\hat{T}-\text { odd }}$ and $a_{C P}^{\hat{T}-\text { odd }}$ that are sensitive to $P$ and $C P$ violation respectively [32]. These asymmetries are measured in bins in two schemes. The first bins across the Dalitz space of the 4-body decay, the second as a function of angle between the decay plane of the 
proton and the fastest $\pi^{-}$compared to the decay plane of the two other pions. The resulting asymmetry is shown in Fig. 7. A permutation test of the $C P$ violating effect concludes the significance of the $C P$ violation is $3.3 \sigma$ and thus represents the first evidence of $C P$ violation in baryons. An alternative description is available in these proceedings [33].
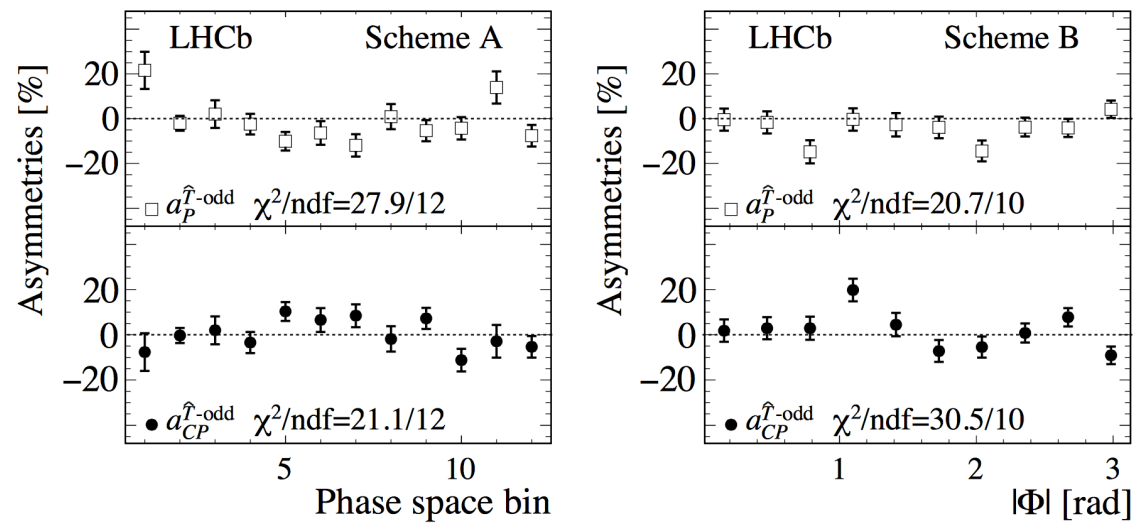

Figure 7: The results for each of the two binning schemes described in the text. The asymmetries $a_{P}^{\hat{T}-\text { odd }}$

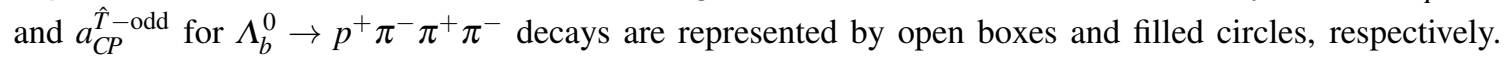
The values of the $\chi^{2} /$ ndf are quoted for the $P$ - and $C P$-conserving hypotheses for each binning scheme. Reproduced from [32].

\section{New searches}

The pursuit of a comprehensive understanding of $C P$ violation needs new hadronic modes of $b$-hadrons. A number of new decay modes are reported, albeit without $C P$ violation investigations.

\section{$5.1 B \rightarrow$ charmless}

Searches for two charmless $B_{s}^{0}$ decays are reported by LHCb: $B_{s}^{0} \rightarrow \phi \pi^{+} \pi^{-}$and $B_{s}^{0} \rightarrow \phi \eta^{\prime}$. Both are mediated, in part, by gluonic penguin diagrams with potential sensitivity to the $B_{s}^{0}$ mixingphase, $\beta_{s}$, with an eventual time-dependent analysis. These searches however, are untagged and time-integrated, searching for signals in the $3 \mathrm{fb}^{-1}$ samples from 2011 and 2012. An event yield of $697 \pm 30 B_{s}^{0} \rightarrow \phi \pi^{+} \pi^{-}$candidates are observed with little background. A study of the $\pi^{+} \pi^{-}$ invariant mass distribution in Fig. 8 show that it is dominated by the light strangeless combinations $f_{0}(980), f_{2}(1270)$ and the $f_{0}(1500)$. A total branching fraction is measured:

$$
\mathscr{B}\left(B_{s}^{0} \rightarrow \phi \pi^{+} \pi^{-}\right)=(3.48 \pm 0.23 \pm 0.17 \pm 0.35) \times 10^{-6},
$$

where the third uncertainty is due to the normalisation with respect to $B_{s}^{0} \rightarrow \phi \phi$. Around a third of the $\pi^{+} \pi^{-}$combinations are found in the narrow $f_{0}(980)$ resonance. A dedicated proceedings is available [34].

From Belle, two new signals are presented. The first is and observation of $B_{s}^{0} \rightarrow K^{0} \bar{K}^{0}$ with branching fraction,

$$
\mathscr{B}\left(B_{s}^{0} \rightarrow K^{0} \bar{K}^{0}\right)=\left(19.6_{-5.1}^{+5.8} \pm 1.0 \pm 2.0\right) \times 10^{-6},
$$



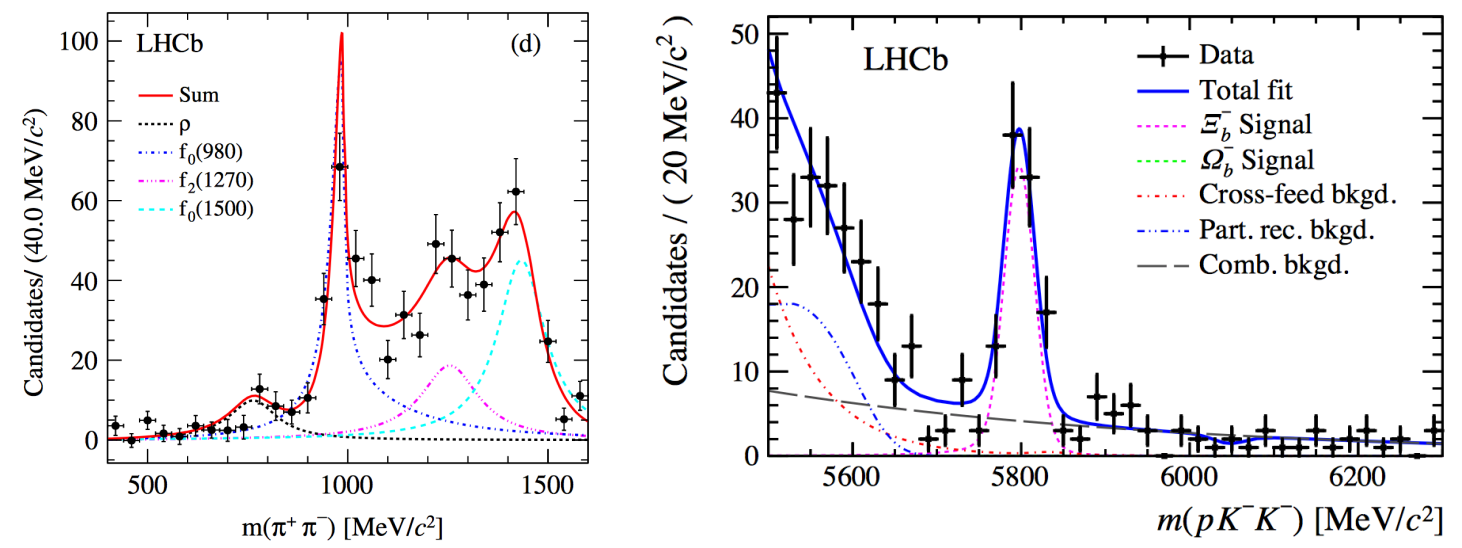

Figure 8: left: background-suppressed invariant mass distribution on $m\left(\pi^{+} \pi^{-}\right)$in $B_{s}^{0} \rightarrow \phi \pi^{+} \pi^{-}$decays. The contributing intermediate $\pi^{+} \pi^{-}$resonances, as deduced by an angular analysis, are shown in the legend. right: Mass distributions for $b$-hadron candidates in the $p K^{-} K^{-}$final state. The $\Xi_{b}$ signal decays are shown with pink dashed lines and partially reconstructed backgrounds are shown with dark dashed lines.

from an analysis of $6.5 \mathrm{M} B_{s}^{0} \bar{B}_{s}^{0}$ pairs accumulated at the $e^{+} e^{-} \rightarrow \Upsilon(5 S)$ resonance. This decay mode is of similar interest as the previously-observed two-body mode, $B_{s}^{0} \rightarrow K^{+} K^{-}$. Evidence of $B^{0} \rightarrow \eta \eta$ is also presented from the analysis of $753 \mathrm{M} B \bar{B}$ pairs accumulated at the $e^{+} e^{-} \rightarrow \Upsilon(4 S)$ resonance with branching fraction,

$$
\mathscr{B}\left(B^{0} \rightarrow \eta \eta\right)=\left(7.6_{-2.3-1.3}^{+2.7+1.4}\right) \times 10^{-7} .
$$

This decay receives contributions from $b \rightarrow u$ tree and $b \rightarrow d$ penguin amplitudes and could contribute to Unitarity angle $\alpha$ measurements at Belle II. A dedicated proceedings is available [31].

\section{$5.2 \Xi_{b} \rightarrow p K^{-} K^{-}$}

The evidence of $C P$ violation in the baryonic sector [32] summarised in Sec. 4.3 motivates the development of hadronic decays of $b$-baryons. $C P$-asymmetry parameters are measured in threebody $\Lambda_{b}^{0}$ baryon decays to $K_{\mathrm{S}}^{0} p \pi^{-}$[35], $\Lambda K^{+} K^{-}$and $\Lambda K^{+} \pi^{-}$[36] and found to be consistent with zero. In the search of $C P$-violation effects the $p K^{-} K^{-}$final state is reconstructed by LHCb. The decay $\Xi_{b} \rightarrow p K^{-} K^{-}$proceeds via the combination of $b \rightarrow u$, colour-suppressed tree decays and $b \rightarrow s$ penguin decays. LHCb observe $83 \pm 10$ events of this mode, the data is shown in Fig. 8 [37].

\subsection{Baryonic $B$ decays}

Branching fraction of charmless baryonic $B$ decays are $\mathscr{O}\left(10^{-6}\right)$ and have been observed in several modes of $B^{+}$and $B^{0}$ mesons, but not previously in $B_{s}^{0}$ decays. As two-body baryonic decays are suppressed with respect to multibody decays, $\mathrm{LHCb}$ reports a search for baryonic $B_{s}^{0}$ decays into three-body final state, $B_{s}^{0} \rightarrow p \bar{\Lambda} h^{-}$where $h \in\{\pi, K\}$ [38]. The result of this successful search is an observation of $260 \pm 21 B_{s}^{0} \rightarrow p \bar{\Lambda} K^{-}$decays. The distribution of these events is investigated on the Dalitz plane. No significant intermediate structure is seen except for a threshold enhancement at low $m(p \bar{\Lambda})$, see Fig. 9. A branching fraction for this baryonic decay of the $B_{s}^{0}$ meson is quoted, 

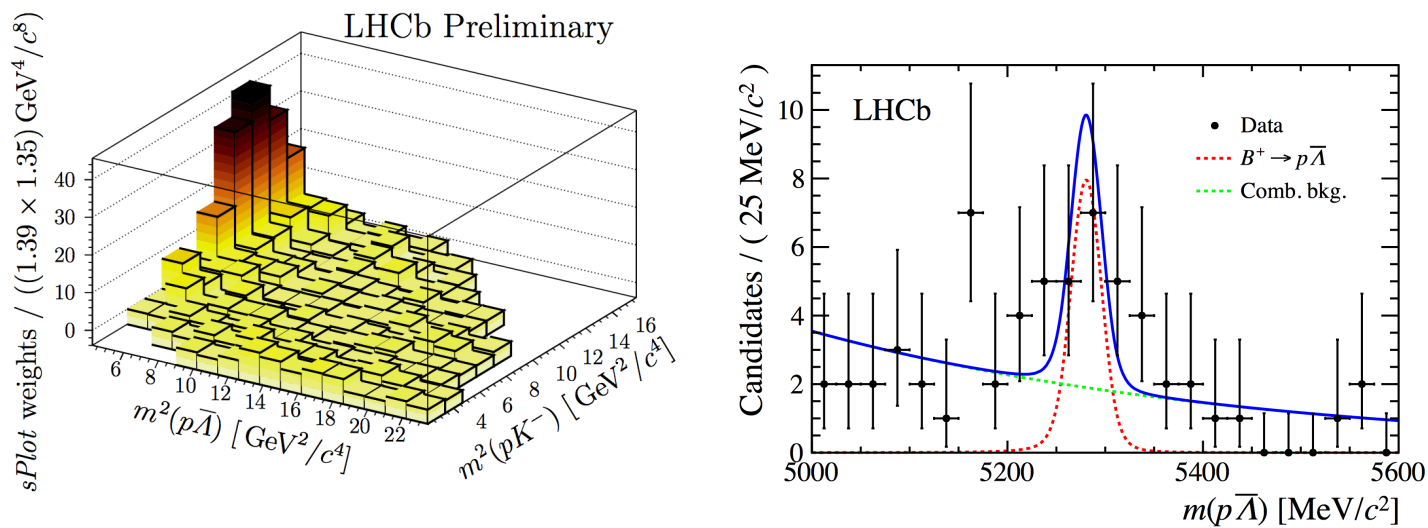

Figure 9: left: Dalitz plot of $p \bar{\Lambda} K^{-}$combinations consistent with a $B_{s}^{0}$ mass, a thershold enhancement is observed but no resonances. right: Invariant mass distribution of $p \bar{\Lambda}$ combinations that exhibit evidence of the $B^{+} \rightarrow p \bar{\Lambda}$ decay mode.

$$
\mathscr{B}\left(B_{s}^{0}+\bar{B}_{s}^{0} \rightarrow p \bar{\Lambda} K^{-}\right)=\left(5.48_{-0.80}^{+0.82} \pm 0.85\right) \times 10^{-6} .
$$

These final states have been postulated to manifest a $\mathscr{O}(10 \%) T$ violation [39] and should be investigated with larger datasets.

LHCb also report the first evidence $(4.1 \sigma)$ of the rare two-body baryonic decay mode, $B^{+} \rightarrow$ $p \bar{\Lambda}$. This mode is analogous to the two-body mesonic decay $B^{+} \rightarrow K \pi$ but with a different arrangement of quarks in the final state. $B^{+} \rightarrow p \bar{\Lambda}$ is expected to be dominated by a $b \rightarrow s$ penguin transition with contribution from suppressed $b \rightarrow u$ tree and annihilation processes. That collaboration report a branching fraction of,

$$
\mathscr{B}\left(B^{+} \rightarrow p \bar{\Lambda}\right)=\left(2.4_{-0.8}^{+1.0} \pm 0.3\right) \times 10^{-7},
$$

normalising to $B^{+} \rightarrow K_{\mathrm{S}}^{0} \pi^{+}$decays, assuming half of the $K^{0} B$-decay products mix to $K_{\mathrm{S}}^{0}$ mesons. This evidence is reproduced from [40] in Fig. 9. A dedicated proceedings is available [41].

\section{$5.4 B_{c}^{+} \rightarrow D^{0} K^{+}$}

The $B_{c}^{+}$meson is the only meson to contain two heavy flavour quarks of different flavour. It has been observed in a number of decay modes with a $J / \psi$, which is expected from a $b \rightarrow$ $c$ external $W$-emission tree decay. The lifetime of the $B_{c}^{+}$meson has recently been measured, $\tau\left(B_{c}^{+}\right)=0.513 \pm 0.012$ ps [42], which is significantly shorter than the lifetimes of the other $B$ mesons which all lie in the interval $1.50-1.64 \mathrm{ps}$ [43]. The shorter lifetime is attributed to a large fraction $(\sim 60 \%)$ of its partial width being due to Cabibbo favoured $c \rightarrow s$ transitions of the charm quark, with only $\sim 20 \%$ from cross-generation $\bar{b} \rightarrow \bar{c}$ transition. The first observation of a charm quark decay in a $B_{c}^{+}$meson was $B_{c}^{+} \rightarrow B_{s}^{0} \pi^{+}$with an approximate branching fraction of around $10 \%$ [44]. The third classification of $B_{c}^{+}$decay, with around $10 \%$ of the total width, is the annihilation topology. Annihilation decays are unambiguously probed in $B$ mesons with searches for final states that contain no quark from the initial state; in the case of $B_{c}^{+}$decays, this is charmless decays. LHCb has conducted searches for charmless $B_{c}^{+}$decays in the $p \bar{p} \pi^{+}$final state 
but found no signal, setting an upper limit on $\sigma\left(B_{c}^{+}\right) / \sigma\left(B^{+}\right) \times \mathscr{B}\left(B_{c}^{+} \rightarrow p \bar{p} \pi^{+}\right)<3.6 \times 10^{-8}$ for $m(p \bar{p})<2.85 \mathrm{GeV} / c^{2}$ where $\sigma$ symbolises the production cross section [45]. A similar search is conducted in the $\bar{K}^{* 0} K^{+}$final state where a $2.4 \sigma$ excess is reported with $\sigma\left(B_{c}^{+}\right) / \sigma\left(B^{+}\right) \times \mathscr{B}\left(B_{c}^{+} \rightarrow\right.$ $\left.\bar{K}^{* 0}\left(\rightarrow K^{-} \pi^{+}\right) K^{+}\right) \sim[0.1,1.7] \times 10^{-8}$ for $m\left(K^{-} \pi^{+}\right)<1.834 \mathrm{GeV} / c^{2}$. The low values of these quantities indicates a small decay constant for $B_{c}^{+}$mesons and, in turn the annihilation topology. Another decay that may receive a large, though not unique signature from $\bar{b}+c$ annihilation is $B_{c}^{+} \rightarrow D^{0} K^{+}$. At the conference LHCb reported an observation of $B_{c}^{+} \rightarrow D^{0} K^{+}$[46] with with a

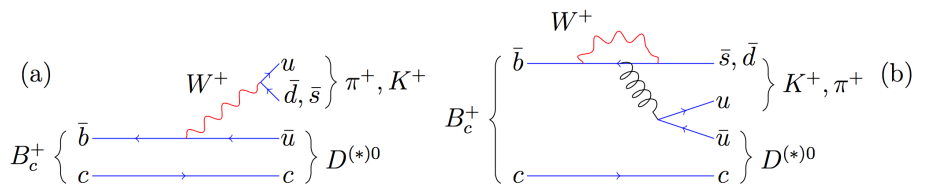

$B_{c}^{+}\left\{\begin{array}{c}\bar{b} \\ \bar{s}, \bar{d} \\ W_{c}^{+}\end{array}\right\} K^{+}, \pi^{+}$
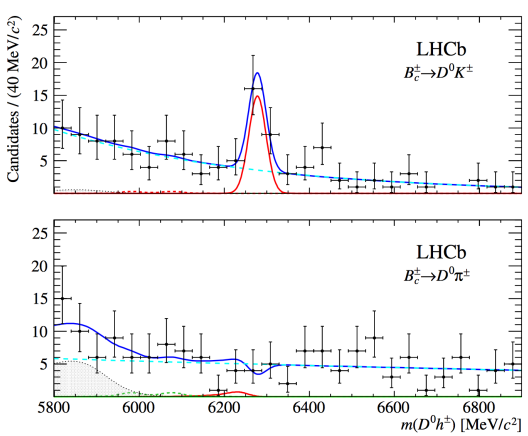

Figure 10: left: Feynman diagrams of processes contributing to $B_{c}^{+} \rightarrow D^{0} h^{+}$decays (a) $b \rightarrow u$ transition, (b) $b \rightarrow s$ penguin, (c) $\bar{b}+c$ annihilation. right: Invariant mass of $m\left(D^{0} K^{+}\right)$and $m\left(D^{0} \pi^{+}\right)$combinations.

branching fraction multiplied by the ratio of cross-sections,

$$
\frac{\sigma\left(B_{c}^{+}\right)}{\sigma\left(B^{+}\right)} \times \mathscr{B}\left(B_{c}^{+} \rightarrow D^{0} K^{+}\right)=\left(9.3_{-2.5}^{+2.8} \pm 0.6\right) \times 10^{-7},
$$

as shown in Fig. 10. The ratio of cross sections is expected to be between 0.003 and 0.011 , implying that the $B_{c}^{+}$branching fraction is $\mathscr{O}\left(10^{-4}\right)$, which is higher than predicted [47].

\section{Conclusions}

Direct $C P$ violation in $B$ decays remains a vibrant topic. With only a quarter of the total LHCb dataset analysed and with Belle II imminent, the interest will continue. The measurement of $\gamma$ is becoming more accurate, and a relative precision similar to that of $\beta$ is a realistic target within the next decade. Multibody charmless decays of $b$-hadrons have a rich phenomenology but the separation of CKM information from the hadronic parameters remains a theoretical challenge. Finally, several new modes have been observed and reported to the conference, and we anticipate measurements of $C P$ asymmetries with them in the future, from the datasets of Belle II and the LHCb upgrade.

\section{Acknowledgements}

The authors would like to express their thanks to the excellent speakers who took the time to join Working Group 5 in Mumbai, to share their thoughts and results. Huge thanks also to the organisers for the invitation to convene, and for the immaculate management of this fascinating and diverse conference. 


\section{References}

[1] M. Gronau and D. Wyler, "On determining a weak phase from $C P$ asymmetries in charged $B$ decays", Phys. Lett. B 265 (1991) 172.

[2] D. Atwood, I. Dunietz and A. Soni, "Improved methods for observing $C P$ violation in $B^{ \pm} \rightarrow K D$ and measuring the CKM phase gamma”, Phys. Rev. D 63 (2001) 036005 [hep-ph/0008090].

[3] A. Giri, Y. Grossman, A. Soffer and J. Zupan, "Determining gamma using $B^{ \pm} \rightarrow D K^{ \pm}$with multibody D decays", Phys. Rev. D 68 (2003) 054018 [hep-ph/0303187].

[4] R. Aaij et al. [LHCb Collaboration], "Measurement of the CKM angle $\gamma$ from a combination of LHCb results", JHEP 1612 (2016) 087 [hep-ex/1611.03076].

[5] F. Machefert, "Measurement of $\gamma$ from $B$ meson decay to $D^{(*)} K^{(*)}$ " in proceedings of "9th International Workshop on the CKM Unitarity Triangle", PoS(CKM2016)113

[6] T. Gershon, "Current challenges and future prospects for $\gamma$ from $B \rightarrow D h h^{\prime}$ decays" in proceedings of "9th International Workshop on the CKM Unitarity Triangle" PoS(CKM2016)115

[7] J. Charles et al. [CKMfitter Group], " $C P$ violation and the CKM matrix: Assessing the impact of the asymmetric $B$ factories”, Eur. Phys. J. C 41 (2005) no.1, [hep-ph/0406184].

[8] M. Bona et al. [UTfit Collaboration], "The 2004 UTfit collaboration report on the status of the unitarity triangle in the standard model”, JHEP 0507 (2005) 028 [hep-ph/0501199].

[9] M. Bona, "Latest results for the Unitary Triangle fit from the UTfit Collaboration" in proceedings of "9th International Workshop on the CKM Unitarity Triangle" PoS(CKM2016)096.

[10] LHCb Collaboration, "Study of the decay $B^{ \pm} \rightarrow D K^{* \pm}$ with two-body $D$ decays", LHCb-CONF-2016-014.

[11] B. Aubert et al. [BaBar Collaboration], "Measurement of $C P$ violation observables and parameters for the decays $B^{ \pm} \rightarrow D K^{* \pm}$, Phys. Rev. D 80 (2009) 092001 [hep-ex/0909.3981].

[12] D. Červenkov, "Belle results for $\phi_{3}$ and prospects from Belle II by 2021" in proceedings of "9th International Workshop on the CKM Unitarity Triangle" PoS(CKM2016)114.

[13] M. Rama, "Effect of $D-\bar{D}$ mixing in the extraction of $\gamma$ with $B^{-} \rightarrow D^{0} K^{-}$and $B^{-} \rightarrow D^{0} \pi^{-}$decays", Phys. Rev. D 89 (2014) no.1, 014021 [hep-ex/1307.4384].

[14] Y. Grossman and M. Savastio, "Effects of $K^{0}-\bar{K}^{0}$ mixing on determining $\gamma$ from $B^{ \pm} \rightarrow D K^{ \pm}$", JHEP 1403 (2014) 008 [hep-ph/1311.3575].

[15] J. Brod, "Electroweak effects in the extraction of the CKM angle $\gamma$ from B $\rightarrow D \pi$ decays", Phys. Lett. B 743 (2015) 56 [hep-ph/1412.3173].

[16] J. Brod and J. Zupan, "The ultimate theoretical error on $\gamma$ from $B \rightarrow D K$ decays", JHEP 1401 (2014) 051 [hep-ph/1308.5663].

[17] J. Brod, A. Lenz, G. Tetlalmatzi-Xolocotzi and M. Wiebusch, "New physics effects in tree-level decays and the precision in the determination of the quark mixing angle”, Phys. Rev. D 92 (2015) no.3, 033002 [hep-ph/1412.1446].

[18] R. Aaij et al. [LHCb Collaboration], "Measurement of $C P$ observables in $B^{ \pm} \rightarrow D K^{ \pm}$and $B^{ \pm} \rightarrow D \pi^{ \pm}$ with two- and four-body D decays", Phys. Lett. B 760 (2016) 117 [hep-ex/1603.08993].

[19] R. Aaij et al. [LHCb Collaboration], "Measurement of the CKM angle $\gamma$ using $B^{ \pm} \rightarrow D K^{ \pm}$with $D \rightarrow K_{\mathrm{S}}^{0} \pi^{+} \pi^{-}, K_{\mathrm{S}}^{0} K^{+} K^{-}$decays", JHEP 1410 (2014) 097 [hep-ex/1408.2748]. 
[20] S. Malde et al., "First determination of the $C P$ content of $D \rightarrow \pi^{+} \pi^{-} \pi^{+} \pi^{-}$and updated determination of the $C P$ contents of $D \rightarrow \pi^{+} \pi^{-} \pi^{0}$ and $D \rightarrow K^{+} K^{-} \pi^{0}$," Phys. Lett. B 747 (2015) 9 [hep-ex/1504.05878].

[21] P. d'Argent et al., "Amplitude Analyses of $D^{0} \rightarrow \pi^{+} \pi^{-} \pi^{+} \pi^{-}$and $D^{0} \rightarrow K^{+} K^{-} \pi^{+} \pi^{-}$Decays", [hep-ex/1703.08505].

[22] S. Harnew, https://github.com/samharnew/HyperPlot.git.

[23] Resmi P.K., "Quantum-correlated measurements of $D^{0} \rightarrow K_{\mathrm{S}}^{0} \pi^{+} \pi^{-} \pi^{0}$ and consequences for the determination of $\gamma$ " in proceedings of "9th International Workshop on the CKM Unitarity Triangle" $\operatorname{PoS}(\mathrm{CKM} 2016) 112$

[24] M. Beneke and S. Jager, "Spectator scattering at NLO in non-leptonic B decays: Leading penguin amplitudes”, Nucl. Phys. B 768 (2007) 51 [hep-ph/0610322].

[25] A. Jain, I. Z. Rothstein and I. W. Stewart, "Penguin loops for nonleptonic $B$-decays in the Standard Model: Is there a Penguin Puzzle?”, arXiv:0706.3399 [hep-ph].

[26] G. Bell and T. Huber, "Master integrals for the two-loop penguin contribution in non-leptonic B-decays", JHEP 1412 (2014) 129 [hep-ph/1410.2804].

[27] G. Bell, M. Beneke, T. Huber and X. Q. Li, “Two-loop currentĐcurrent operator contribution to the non-leptonic QCD penguin amplitude”, Phys. Lett. B 750 (2015) 348 [hep-ph/1507.03700].

[28] S. Kränkl, T. Mannel and J. Virto, “Three-body non-leptonic B decays and QCD factorization”, Nucl. Phys. B 899 (2015) 247 [hep-ph/1505.04111].

[29] J. Charles, S. Descotes-Genon, J. Ocariz and A. Pérez Pérez, "Disentangling weak and strong interactions in $B \rightarrow K^{*}(\rightarrow K \pi) \pi$ Dalitz-plot analyses", [hep-ph/1704.01596].

[30] R. Aaij et al. [LHCb Collaboration], "Measurements of $C P$ violation in the three-body phase space of charmless $B^{ \pm}$decays", Phys. Rev. D 90 (2014) no.11, 112004 [hep-ex/1408.5373].

[31] C.-L. Hsu, "Results from B factories in charmless (quasi)-two-body decays and Belle II prospects" 9th International Workshop on the CKM Unitarity Triangle" PoS(CKM2016)106

[32] R. Aaij et al. [LHCb Collaboration], "Measurement of matter-antimatter differences in beauty baryon decays", Nature Phys. (2017) [hep-ex/1609.05216].

[33] A. Merli, "Results from LHCb in charmless b-baryon decays" in proceedings of "9th International Workshop on the CKM Unitarity Triangle" PoS(CKM2016)103

[34] S. Giani, "Results from LHCb in charmless (quasi)-two body B meson decays" in proceedings of "9th International Workshop on the CKM Unitarity Triangle" PoS(CKM2016)107

[35] R. Aaij et al. [LHCb Collaboration], "Searches for $\Lambda_{b}^{0}$ and $\Xi_{b}^{0}$ decays to $K_{\mathrm{S}}^{0} p \pi^{-}$and $K_{\mathrm{S}}^{0} p K^{-}$final states with first observation of the $\Lambda_{b}^{0} \rightarrow K_{\mathrm{S}}^{0} p \pi^{-}$decay”, JHEP 1404 (2014) 087 [hep-ex/1402.0770].

[36] R. Aaij et al. [LHCb Collaboration], "Observations of $\Lambda_{b}^{0} \rightarrow \Lambda K^{+} \pi^{-}$and $\Lambda_{b}^{0} \rightarrow \Lambda K^{+} K^{-}$decays and searches for other $\Lambda_{b}^{0}$ and $\Xi_{b}^{0}$ decays to $\Lambda h^{+} h^{\prime-}$ final states", JHEP 1605 (2016) 081 [hep-ex/1603.00413].

[37] R. Aaij et al. [LHCb Collaboration], "Observation of the decay $\Xi_{b}^{-} \rightarrow p K^{-} K^{-}$, Phys. Rev. Lett. 118 (2017) no.7, 071801 [hep-ex/1612.02244].

[38] The LHCb Collaboration [LHCb Collaboration], "First observation of a baryonic $B_{s}^{0}$ decay", LHCb-CONF-2016-016. 
[39] C. Q. Geng and Y. K. Hsiao, "Direct $C P$ and $T$ Violation in Baryonic $B$ Decays", Int. J. Mod. Phys. A 23 (2008) 3290 doi:10.1142/S0217751X08041992 [hep-ph/0801.0022].

[40] R. Aaij et al. [LHCb Collaboration], "Evidence for the two-body charmless baryonic decay $B^{+} \rightarrow p \bar{\Lambda} ",[$ hep-ex/1611.07805].

[41] E. Rodrigues, "Results from LHCb in charmless baryonic B meson decays" 9th International Workshop on the CKM Unitarity Triangle" PoS(CKM2016)104

[42] R. Aaij et al. [LHCb Collaboration], "Measurement of the lifetime of the $B_{c}^{+}$meson using the $B_{c}^{+} \rightarrow J / \psi \pi^{+}$decay mode”, Phys. Lett. B 742 (2015) 29 [hep-ex/1411.6899].

[43] Y. Amhis et al., "Averages of $b$-hadron, $c$-hadron, and $\tau$-lepton properties as of summer 2016", [hep-ex/1612.07233].

[44] R. Aaij et al. [LHCb Collaboration], "Observation of the Decay $B_{c}^{+} \rightarrow B_{s}^{0} \pi^{+}$,, Phys. Rev. Lett. 111 (2013) no.18, 181801 [hep-ex/1308.4544].

[45] R. Aaij et al. [LHCb Collaboration], "Study of $B_{c}^{+}$decays to the $K^{+} K^{-} \pi^{+}$final state and evidence for the decay $B_{c}^{+} \rightarrow \chi_{c 0} \pi^{+}$, Phys. Rev. D 94 (2016) no.9, 091102 [hep-ex/1607.06134].

[46] R. Aaij et al. [LHCb Collaboration], “Observation of $B_{c}^{+} \rightarrow D^{0} K^{+}$decays”, Phys. Rev. Lett. 118 (2017) no.11, 111803 [hep-ex/1701.01856].

[47] J. Zhang and X. Q. Yu, "Branching ratio and $C P$ violation of $B(c) \rightarrow D K$ decays in the perturbative QCD approach”, Eur. Phys. J. C 63 (2009) 435 [hep-ph/0905.0945]. 